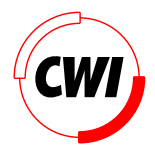

Centrum voor Wiskunde en Informatica

REPORTRAPPORT

Cases, adverbs, situations and events

P. Dekker

Computer Science/Department of Interactive Systems

CS-R9643 1996 
Report CS-R9643

ISSN 0169-118X

CWI

P.O. Box 94079

1090 GB Amsterdam

The Netherlands

$\mathrm{CWI}$ is the National Research Institute for Mathematics and Computer Science. CWI is part of the Stichting Mathematisch Centrum (SMC), the Dutch foundation for promotion of mathematics and computer science and their applications.

SMC is sponsored by the Netherlands Organization for Scientific Research (NWO). CWI is a member of ERCIM, the European Research Consortium for Informatics and Mathematics.

Copyright @ Stichting Mathematisch Centrum P.O. Box 94079, 1090 GB Amsterdam (NL) Kruislaan 413, 1098 SJ Amsterdam (NL) Telephone +3120 5929333 Telefax +3120 5924199 


\title{
Cases, Adverbs, Situations and Events
}

\author{
Paul Dekker \\ CWI, P.O. Box 94079, 1090 GB Amsterdam, The Netherlands \\ ILLC, Nieuwe Doelenstraat 15, 1012 CP Amsterdam, The Netherlands
}

Cats are nice. (...) because cats never overlap.

(David Lewis, Parts of Classes, p. 22)

\begin{abstract}
In this paper we discuss two approaches to adverbial quantification, the so-called 'bound variable approach' and the 'situation-based approach', and we address the suggestion that has been made in the literature that the latter reduces to the former as soon as the underlying structure of situations has been characterized at the required level of detail. We will first show what constraints on situation structures are needed in order for the situation-based approach to produce the results of the bound variable approach. Then we will argue that the required constraints are at odds with the philosophy underlying the situation based approach. This result points at a principled difference between the two approaches, and this, theoretical, difference can be used as a heuristic to find empirical support for any of the two approaches.
\end{abstract}

AMS Subject Classification (1991): 03B65, 68S05, 92K20.

Keywords \&f Phrases: Dynamic Semantics, Situation Theory, Mereology.

Note: The research for this paper was supported by the ESPRIT Basic Research project 6852 (DYANA) and the LRE Project 62-01 (FraCaS).

\section{INTRODUCTION}

In [Lewis 1975] examples are discussed of the form:

(1) Sometimes / usually / always, if a Dutchman smells a bargain, he goes for it.

Lewis points out that the quantifying adverbs sometimes / usually / always in fact quantify over the 'cases' that verify the restrictive clause. The cases that verify the restriction a Dutchman smells a bargain are conceived of as pairs consisting of a Dutchman and a bargain he smells, and the respective quantifiers quantify over these cases. If the adverb is sometimes, the sentence says that some pairs consisting of a Dutchman and a bargain he smells are pairs of which the first element purchases the second. If always is the head, the sentence tells us that every pair of a Dutchman and a reeking bargain stands in the purchase relation. With usually, it can be stated that most such pairs stand in this relation. Put more generally, adverbs of quantification are taken to quantify over the sequences of individuals satisfying the conditions imposed upon the values of indefinite noun phrases in the restrictions of the adverbs.

With Lewis' approach to adverbial quantification a whole family of analyses can be associated (among which, e.g., [Heim 1982, Root 1986, Kadmon 1990, Groenendijk and Stokhof 1991b, Chierchia 1992, Dekker 1993]) and in the sequel this family of analyses will be referred to as the 'Bound Variable Approach' ( $B V A$, 'Free Variable Approach' would do equally well, for that matter). Another, alternative, family of analyses can be referred to as 'Situation-based approach' (henceforth $S b A$ ). According to analyses of this kind the domains of adverbial quantification consist of the 'minimal situations' which support the adverbs' restrictions (where the notion of a situation must be understood in the 
sense of [Kratzer 1989]). Thus, the example above expresses quantification over 'minimal situations' in which a Dutchman smells a bargain, and it can be used to state that some / most / all such situations are (part of) situations in which the Dutchman goes for the bargain. Analyses of this kind can be found in, among others, [Berman 1987, Chierchia 1988, Heim 1990, van Eijck and de Vries 1991, de Swart 1991, Fintel 1995].

A major problem of, or challenge for, the $S b A$ is that it doesn't give any account of the inferential properties of adverbially quantified srtuctures, as long as it does not specify what the underlying structure of situations is. For instance, what the $S b A$, as it is often presented, fails to account for is that many people judge (3) a logical consequence of (2):

(2) If a cat falls down, it always lands on its feet.

(3) Every cat who falls down lands on its feet.

However, if the structure of situations underlying the $S b A$ is not constrained in any way, then the adverbially quantified construction may come out true, and the corresponding adnominally quantified construction false. For even if there are many cats which fall down, and which do not land on their feet, there may fail to be minimal situations in which a cat falls down, and, hence, the adverbial quantification may be vacuous. Another example of an (unwanted) non-validity can be found in:

(4) If a kid has had primary education in Amsterdam, it never knows the national anthem

(5) No kid who has had primary eduction in Amsterdam knows the national anthem.

Example (4) is false if it many kids who have had primary education perfectly well know the national anthem. Still, the adverbially quantified statement may be - trivially - true, because there happen to be no minimal situations in which a kid has had primary education in Amsterdam.

It will be clear that for the discussed inferences not to come out valid, we have to bring up possible structures of situations which, although not excluded beforehand, can be conceived to be rather 'deviant' from some intuitive conception of situations, and, hence, should not be taken into consideration. As a matter of fact, situation-based analyses have often been backed up by such calls on intuitions, e.g., that if there is a woman living with a cat, then there is a minimal situation in which only this woman only lives with only this cat. In general the literature on the subject is full of such suggestions about a 'casy', or case-like, appearance of minimal situations (cf., e.g., [Chierchia 1988, Heim 1990, Lappin and Francez 1994]).

The intention of this paper is to flesh out the suggested impression of a 'casy-ness' of the $S b A$ on a formal level. The felt suggestion is that the $S b A$ and the $B V A$ produce the same results, as soon as the structure of situations underlying the $S b A$ has been sufficiently characterized at the required level of detail. To substantiate this we will sort out what a situation structure must be like in order to make it behave like a structure of cases. However, when this is achieved, it will appear that the constraints which have to be imposed on the situation structures are at odds with their background philosophy. Thus, we find there to be a principled difference between the two approaches. The two are bound to make different empirical predictions.

For this reason, the paper concludes with an investigation of the kinds of cases where the two approaches work out differently. It is then argued that in these cases in fact none of the two is really adequate. Instead, the inspected data are taken to motivate a conception of the notion of a situation as that of an eventuality (as adopted in, among others, Davidson, Link, Bach, Krifka). Following [Chierchia 1992] and, recently, [Jäger 1995, Milward 1996], a combined dynamic and situation- or eventuality-based approach is eventually argued for. 


\section{Bound Variable Approach}

According to the $B V A$, adverbs of quantification like 'always', 'sometimes', 'never', 'usually', ... are taken to quantify over variable assignments. In Lewis it is said that a sentence like (6):

(6) ADVERB, if a farmer owns a donkey, he beats it.

must be taken in the logician's idiom as something which sounds like $A D V E R B$, if (some) $x$ is a farmer, (some) y a donkey, and $x$ owns $y$, then $x$ beats $y$, and this in its turns has to be analyzed as involving quantification over possible assignments of values to the variables $x$ and $y$. Under this analysis, example (6) is true if all assignments of values to the variables $x$ and $y$ under which the value of $x$ is a farmer who beats a donkey which is the value of $y$, are assignments under which the value of $x$ beats the value of $y$.

Although the analysis of many examples can be stated in terms of total assignment, the adverbials quantifiers in general must be taken to quantify over certain alternatives of variable assignmentsthis in view of adverbs such as 'often', 'twice', 'more_then_once'-, and these alternatives can be best viewed as partial variable assignments (cf., e.g. [Dekker 1995]). Relative to a domain $E$ of individuals, and subsets $X$ of the set $V$ of variables, the relevant domains of quantification can be defined as follows:

Definition 1 For finite $X \subseteq V: D o Q_{B V A}^{X}=E^{X}$

Assuming some minimal language of predicate logic, without individual constants and identity, we can define an interpretation function which generates the appropriate subsets of the domains of adverbial quantification. The interpretation defined below is a dynamic predicate logical version of Heim's file change semantics. This semantics is dynamic since it can be seen as changing the assignment parameter. In general, relative to a partial variable assignment $i$, the interpretation of a formula yields a set of (possibly alternative) variable assignments as possible results. For instance, the interpretation of a conjunction $\phi \wedge \psi$ relative to $i$ is the set of assignments which are a possible result of interpreting $\psi$ relative to an assignment which is a possible result from interpreting $\phi$ relative to $i$. This encodes the 'dynamic' idea that the interpretation of $\phi \wedge \psi$ involves the 'successive' interpretation of $\phi$ and $\psi$, in that order (cf., [Groenendijk and Stokhof 1991a]).

The specific interpretation function used here also incorporates a notion of felicity in the sense of [Heim 1982], and this function is, therefore, partial. It renders the interpretation of an atomic formula $R x_{1} \ldots x_{n}$ undefined relative to a partial assignment $i$ iff $i$ itself is undefined for any of the variables $x_{1} \ldots x_{n}$; the interpretation of an existentially quantified formula $\exists x \phi$ is undefined relative to partial assignment $i$ iff $i$ itself is defined for $x$ or if $\phi$ is undefined for an extension of $i$ to $x$. A negation $\neg \phi$ is undefined relative to $i$ iff $\phi$ is; and a conjunction $\phi \wedge \psi$ is undefined relative to $i$ iff $\phi$ is, or if $\psi$ is undefined for any of the assignments which result from interpreting $\phi$ relative to $i$. Throughout, we will assume definedness, which, by the way, can be fully characterized syntactically. (For relevant discussion, cf., [Dekker 1995]).

Now, let $M=\langle E, F\rangle$ be an ordinary first order model, let $i, j, k$ be partial variable assignments, and let $i \leq_{x} k$ say that $k$ extends $i$ with an assignment to $x$. Then the interpretation $\llbracket \phi \rrbracket_{M, i}$ of $\phi$ relative to $M$ and $i$ (if defined at all), is specified as follows:

\section{Definition 2 (Dynamic Semantics)}

$$
\begin{array}{ll}
\llbracket R x_{1} \ldots x_{n} \rrbracket_{M, i} & =\left\{j \mid i=j \&\left\langle i\left(x_{1}\right), \ldots, i\left(x_{n}\right)\right\rangle \in F_{M}(R)\right\} \\
\llbracket \neg \phi \rrbracket_{M, i} & =\left\{j \mid i=j \& \llbracket \phi \rrbracket_{M, i}=\emptyset\right\} \\
\llbracket \exists x \phi \rrbracket_{M, i} & =\left\{j \mid \exists k: i \leq_{x} k \& j \in \llbracket \phi \rrbracket_{M, k}\right\} \\
\llbracket \phi \wedge \psi \rrbracket_{M, i} & =\left\{j \mid \exists k: k \in \llbracket \phi \rrbracket_{M, i} \& j \in \llbracket \psi \rrbracket_{M, k}\right\} \\
\llbracket A(\phi)(\psi) \rrbracket_{M, i} & =\left\{j \mid i=j \&[A]\left(\left\{j \mid j \in \llbracket \phi \rrbracket_{M, i}\right\}\right)\left(\left\{j \mid \llbracket \psi \rrbracket_{M, j} \neq \emptyset\right\}\right)\right\}
\end{array}
$$


where $[A]$ is the usual binary interpretation associated with adverb $A$.

Let us quickly see what the above definition amounts to:

(7) Always if a Dutchman smells a bargain, he goes for it. $A(\exists x(D x \wedge \exists y(B y \wedge S x y)))(G x y)$

The sentence is satisfied in $M$ relative to an assigment $i$ iff $G x y$ is satisfied in $M$ relative to all extensions $j$ of $i$ in $\llbracket \exists x(D x \wedge \exists y(B y \wedge S x y)) \rrbracket_{M, i}$. This is the case iff under every assignment of values $d$ and $d^{\prime}$ to $x$ and $y$, we find that if $d$ is a Dutchman, $d^{\prime}$ a bargain, and if $d$ smells $d^{\prime}$, then $d$ goes for $d^{\prime}$.

Assuming our adverbial quantifiers to observe the constraints of conservativity and quantity, quantification over the domains of partial variable assignments can be seen to amount to quantification over domains consisting of Lewisian cases, i.e., tuples of individuals. For for finite $X \subseteq V$ and $n=|X|$ :

Observation 1 there is a bijection $\pi$ from $D o Q_{B V A}^{X}$ to $E^{n}$

Proof. Let $|X|=n$, and let $x_{1}, \ldots x_{n}$ be an enumeration of $X$. Then $\pi$ can be defined such that for $i \in D o Q_{B V A}^{X}: \pi(i)=\left\langle i\left(x_{1}\right), \ldots, i\left(x_{n}\right)\right\rangle\left(\in E^{n}\right)$. Its inverse $\pi^{-1}$ is given by $\pi^{-1}\left(\left\langle d_{1}, \ldots, d_{n}\right\rangle\right)=$ $\left\{\left\langle x_{i}, d_{i}\right\rangle \mid i \leq n\right\}$. End of Proof.

Using the mentioned constraints we find that:

$$
\begin{aligned}
& {[A]\left(\left\{j \mid j \in \llbracket \phi \rrbracket_{M, i}\right\}\right)\left(\left\{j \mid \llbracket \psi \rrbracket_{M, j} \neq \emptyset\right\}\right) \text { iff (by conservativity) }} \\
& {[A]\left(\left\{j \mid j \in \llbracket \phi \rrbracket_{M, i}\right\}\right)\left(\left\{j \mid j \in \llbracket \phi \rrbracket_{M, i} \text { and } \llbracket \psi \rrbracket_{M, j} \neq \emptyset\right\}\right) \text { iff (by quantity) }} \\
& {[A]\left(\left\{\pi(j) \mid j \in \llbracket \phi \rrbracket_{M, i}\right\}\right)\left(\left\{\pi(j) \mid j \in \llbracket \phi \rrbracket_{M, i} \text { and } \llbracket \psi \rrbracket_{M, j} \neq \emptyset\right\}\right)}
\end{aligned}
$$

In fact this is what had been assumed in the first place. Quantification over assignments does not seem to make good sense unless it boils down to quantification over (constructions out of) individuals in the real world. Since, moreover, for any $j \in \llbracket \phi \rrbracket_{M, i}$, in the present system of interpretation we find that $j$ is an extension of $i$ with a set of variables specific for $\phi$, viz., the set of variables 'introduced' by $\phi$, we find (by quantity again), that adverbial quantification is in effect over the tuples of values of variables introduced by the restriction of the adverb. (For those who are interested it may be added that it is straightforward to extend the analysis to asymmetric and adnominal quantification, cf., [Dekker 1995]).

As a matter of fact, Lewisian adverbial quantifiers can more in general be taken to range over tuples consisting of all kinds of entities. Let us assume we have a many-sorted predicate logic, that $\Sigma$ is a set of sorts, and for $\sigma \in \Sigma, D_{\sigma}$ the domain of entities of sort $\sigma$. Then the intended domains of quantification can be more generally characterized as:

(8) $D o Q_{B V A}^{\prime}=\left\{D_{\sigma_{1}} \times \ldots \times D_{\sigma_{n}} \mid \sigma_{1}, \ldots, \sigma_{n} \in \Sigma \& n \in \mathcal{N}\right\}$

Thus we can have all the kinds of sorts one may want to quantify over: individuals, possible worlds, events, time intervals, pigeon holes, and: situations. Trivially, then, the $B V A$ subsumes the $S b A$.

\section{Situation Theory}

According to several proposals which fit in the $S b A$, adverbs of quantification quantify over situations of the type of Angelika Kratzer's situation theory. In this section we first investigate to some extent the specific kind of structures that have been proposed. Kratzer's structures of situations can be defined as follows: 
Definition 3 (Situation structures) $K=\langle S, \sqsubseteq\rangle$ consists of a non-empty set of possible situations $S$, possibly with a subset of individuals $A \subset S$, and a part-whole relation $\sqsubseteq$ which partially orders $S$. (So, $\sqsubseteq$ is reflexive, transitive and antisymmetric.) Every situation $s$ is assumed to be part of a unique biggest situation, $w_{s}$, called $s$ 's possible world. We will say that two situations $s$ and $s^{\prime}$ overlap, $s O s^{\prime}$, iff they have a common (non-empty) part, i.e., iff there is a (non-empty) $s^{\prime}$ such that $s^{\prime \prime} \sqsubseteq s$ and $s^{\prime \prime} \sqsubseteq s^{\prime}$. If $s^{\prime} \sqsubseteq s$ and $s^{\prime} \neq s$ we will say that $s^{\prime}$ is a proper part of $s, s^{\prime} \sqsubset s$. A situation is an atom iff it has no proper parts.

It is generally assumed that structures of situations (or events) are closed under sums:

Definition 4 (Summation) For any non-empty set of situations $P, s$ is a sum of $P$ iff every $s^{\prime}$ in $P$ is part of $s$, and if, for any $s^{\prime \prime}$, every $s^{\prime}$ in $P$ is part of $s^{\prime \prime}$, then $s$ is part of $s^{\prime \prime}$. A structure $\langle S, \sqsubseteq\rangle$ is closed under sums iff every subset $P$ of $S$ has a sum.

By the definition of sums and by antisymmetry it follows that if a set of situations $P$ has a sum, then it has a unique sum $\bigoplus P$. As is common practice, we will write $s \oplus s^{\prime}$ for the sum $\bigoplus\left\{s, s^{\prime}\right\}$ of two situations $s$ and $s^{\prime}$.

There is a typical consequence of assuming closure under sums, which sometimes goes unnoticed, viz., that it entails that there exists one world only, viz., $\bigoplus S$. For the world $w_{s \oplus s^{\prime}}$ of the sum of $s$ and $s^{\prime}$ must be the (one and only) world of both $s$ and $s^{\prime}$. So if any two situations have a sum, they are part of the same world, and if all sets of situations have a sum, there exists only one world. As a matter of fact, for the purposes of this paper this is not an unwanted consequence, because our purposes are entirely extensional. Notice that if, on later extensions, we do want to incorporate modality, then either we have to restrict the domain of summation to sets of situations from one and the same world, as probably should be in accordance with Kratzer's conception of the structure of situations, or we must allow for the possibility that situations are entities which can be part of different worlds (as in, for instance, [Landman 1992, Bartsch 1995]).

In the literature on situations, events and plurals, sums are often conceived of as being built up from their parts. This idea can be stated by means of the following formal constraint:

Definition 5 (Generation) If for all $s^{\prime} \sqsubseteq s: s^{\prime} O t$, then $s \sqsubseteq t$

This constraint subtly says that two situations which are composed of the same parts are identical. That is, if a structure satisfies generation, then:

Observation 2 If $s$ is not itself an atom, then $s=\bigoplus\left\{s^{\prime} \mid s^{\prime} \sqsubset s\right\}$.

Proof. By, for all $s^{\prime} \sqsubseteq s$, there is an $s^{\prime \prime}$ in $\left\{s^{\prime \prime \prime} \mid s^{\prime \prime \prime} \sqsubset s\right\}$ such that $s^{\prime} O s^{\prime \prime}$, so, by transitivity and the definition of $\bigoplus, s^{\prime} O \bigoplus\left\{s^{\prime} \mid s^{\prime} \sqsubset s\right\}$. By generation we then find that $s \sqsubseteq \bigoplus\left\{s^{\prime} \mid s^{\prime} \sqsubset s\right\}$. Conversely, all $s^{\prime}$ in $\left\{s^{\prime} \mid s^{\prime} \sqsubset s\right\}$ are part of $s$, so by the definition of $\bigoplus$ we find that $\bigoplus\left\{s^{\prime} \mid s^{\prime} \sqsubset s\right\} \sqsubseteq s$. Antisymmetry then gives us that $s=\bigoplus\left\{s^{\prime} \mid s^{\prime} \sqsubset s\right\}$. End of proof. Here we see that generation, indeed, tell us that every non-atomic situation equals the sum of its (proper!) parts: the same parts generate the same sums.

The notion of generation gets a bit more concrete in case we are dealing with atomic structures. A structure of situations is called atomic iff every situation has an atomic part. Let us use $A(s)$ to indicate that $s$ is an atom. Then the generation constraint gives us that, for atomic $\langle S, \sqsubseteq\rangle$ : 
Observation 3 If $\{a \sqsubseteq s \mid A(a)\} \subseteq\{b \sqsubseteq t \mid A(b)\}$, then $s \sqsubseteq t$

Proof. Since, by the assumption of atomicity, every part of $s$ has an atomic part, which is also an (atomic) part of $t$, it follows that every part of $s$ overlaps with $t$. Hence, by generation, $s$ is part of $t$. End of proof. Now we see that in atomic structures which satisfy generation, the part_of relation between sums can be defined in terms of their atomic parts. Notice, however, that our notion of generation also applies to non-atomic structures.

Generation can also be defined in a negative way. By contraposition, the constraint tells us that if $s$ is not a part of $t$, then $s$ has a part which does not overlap with $t$. Upon this formulation the constraint can be easily seen to entail Krifka's constraint called 'witness element'. Krifka's constraint requires that if $t \sqsubset s$ (and, hence, $s \nsubseteq t$ ), then $s$ has a part which has no overlap with $t$. In a slogan-like way, upon this reformulation the constraint says that different sums are made up of different parts.

Another notion often employed with respect to algebraic structures of the kind discussed here is that of 'freedom'. (We may have to note that the terms 'freedom' and 'generation' as they are used here correspond to those in [Landman 1989, Lønning 1996], but that what we call 'generation', is called 'freedom' in [van Benthem 1983].) For instance, if we take the sum of two atoms this is required not to force along another atom. As Kai von Fintel has put it: "(...), there is a situation that contains all and only my left earlobe, the square root of 2, and Brutus stabbing Caesar" [Fintel 1995, P. 3] ( $\backslash$ emphasis mine, PD). The general idea is that any part of the sum of any set of situations $P$ is made up of parts of the elements of $P$. This can be formulated as follows:

Definition 6 (Freedom) Let $P$ be any non-empty set of situations. Then, if $s \sqsubseteq \bigoplus P$, then there is an $s^{\prime}$ in $P$ such that $s O s^{\prime}$

By transitivity, if $s \sqsubseteq \bigoplus P$, then all parts of $s$ are required to overlap an element in $P$. If we apply this contraint to a temporal structure, it as it were says that the sum of yesterday and tomorrow is required not to include today. Among other things, freedom ensures that for any sets $X$ and $Y$ of atoms in $S$, that:

Observation 4 If $\bigoplus X \sqsubseteq \bigoplus Y$, then $X \subseteq Y$.

Proof. Assume $\bigoplus X \sqsubseteq \bigoplus Y$, and take any $s \in X$. Then $s \sqsubseteq \bigoplus X \sqsubseteq \bigoplus Y$. By freedom, $\exists s^{\prime} \in Y$ : $s O s^{\prime}$, and since $s$ and $s^{\prime}$ are atoms this must be $s$ itself. Hence, $s \in Y$. End of proof. So freedom in a sense says that the same sums have the same parts, or, equivalently, that different parts make different sums. From this constraint also the following principle follows, a generalization of Krifka's constraint labeled 'partition'. Let, for any non-empty set of situations $X, P(X)$ be the closure $\left\{s^{\prime} \sqsubseteq s \mid s \in X\right\}$ of $X$ under the part_of relation. Under the assumption of freedom, then, for non-empty $X$ :

Observation $5 s \sqsubseteq \bigoplus X$ iff there is a subset $Y \subseteq P(X): s=\bigoplus Y$.

Proof. Right-to-left: Clearly, if $s$ is the sum of parts of elements of $X$ then $s$ is part of the sum of $X$. Left-to-right: Let $Y=\left\{s^{\prime} \sqsubseteq s \mid \exists s^{\prime \prime}: s^{\prime} \sqsubseteq s^{\prime \prime}\right\}$. Clearly, $\bigoplus Y \sqsubseteq s$. Now take any $s^{\prime} \sqsubseteq s(\sqsubseteq \bigoplus X)$. By freedom, $s^{\prime}$ overlaps with an element of $X$, so $s^{\prime}$ has a part $s^{\prime \prime}$ which is part of an element of $X$. By the definition of $Y, s^{\prime \prime} \in Y$, and since $s^{\prime} O s^{\prime \prime}, s^{\prime} O \bigoplus Y$. Since this holds for any $s^{\prime} \sqsubseteq s$, generation 
gives $s \sqsubseteq \bigoplus Y$, and antisymmetry $s=\bigoplus Y$. End of Proof. Generation and freedom are quite strong constraints indeed. They make our structure collapse into a bottom-less complementary lattice. For, with generation and freedom, as long as $s$ is not part of $t$, then $t$ has a proper complement in $s$, defined as $s \backslash t=\bigoplus\left\{s^{\prime} \sqsubseteq s \mid \neg s^{\prime} O t\right\}$.

Now we have considered possible structures of situations to some extent, we may turn to their use in an analysis of adverbial quantification. In the remainder of this paper, we will assume our structures to be free and closed under summation. We do not (need to) impose the constraint of generation. This means that the structures we use just observe the laws of mereology, as they have originally been proposed by Stanislaw Leśniewski at the beginning of this century, and as simplified in, among others, [Tarski 1956]. Lewis takes mereology to be "perfectly understood, unproblematic, and certain", cf., [Lewis 1991, p. 72ff].

\section{Situation-Based Approach}

Let us now turn to a rudimentary (Kratzerian) situation semantics. The basic idea of the $S b A$ is that adverbs of quantification quantify over the 'minimal' situations which satisfy the restriction of the adverb. According to this idea a sentence Usually, if a cat falls down, it lands on its feet is analyzed as stating that most minimal situations in which a cat falls down have an extension where you find that the cat lands on its feet. In this section we will present a most simple formal elaboration of this idea in the form of a toy situation semantics for the predicate logical language employed above.

We will define a semantics for the language of predicate logic according to which formulas are interpreted as persistent sets of verifying situations. (A persistent set of situations $P$ is a set closed of under the has_as_part-relation: if $s \in P$ and $s \sqsubseteq s^{\prime}$, then $s^{\prime} \in P$.) This is as in [Kratzer 1989], but for the fact that we restrict attention to situations which are part of one world, not situations from different possible worlds. As a consequence false formulas are interpreted as the empty set of situations. We furthermore make a couple of simplifying assumptions.

As we have seen above indefinites or existential quantifiers are the main source of structure in the domains of adverbial quantification according to the $B V A$. Now, although much attention on this topic in the literature has been devoted to anaphoric relationships between such terms in the restriction of a quantifying adverb and pronouns in its nuclear scope, we will skip this matter in the sequel. The reason is, that, first, the interpretation of pronouns in a situation-based framework is not a settled issue, and, second, it is not relevant for our immediate concerns. Here we are primarily concerned with (comparing) the structures of the domains of adverbial quantification induced by clauses in the restriction of the adverb. For this reason we can make the simplifying assumption that no free variable must be conceived of as anaphorically related to any preceding existential quantifier, and-in terms of the dynamic semantics - that every syntactically free variable is also semantically free.

In the following definition, the (persistent) interpretation of atomic formulas employs a notation $s^{\prime}==_{M, g} A t$, to be understood as saying that $s^{\prime}$ satisfies $A t$ relative to $M$ and $g$, which will be the case if $A t$ denotes a part of $s^{\prime}$. An atomic formula will be taken to denote a situation iff it is true. For the moment it is left unspecified how this denotation comes about. For, as we will see, how we think about this relation relates to how we want the domains of quantification to be individuated. The clauses dealing with negation, existential quantification, conjunction, and adverbial quantification are relatively simple. Interpretation is defined relative to ordinary (total) variable assignments and to a model like the one we used in the $B V A$. In the clause for adverbially quantified statements we let, for any set of situations $P, P^{m}$ indicate the set of minimal elements of $P$, i.e., $\left\{s \in P \mid \neg \exists s^{\prime} \in P: s^{\prime} \sqsubset s\right\}$.

\footnotetext{
${ }^{1}$ A mereology can be based on a primitive part-whole relationship and a notion of fusion according to which something is a fusion of some things iff (i) it has all of them as parts and (ii) each of its parts overlaps with one of them. The only further stipulations are that the part-whole relationship is transitive, that fusion is idempotent $(x=\bigoplus\{x\})$, and that there exists a unique fusion of any number of things. Upon these stipulations a mereological structure can be seen to be a partial order (so, also reflexive, and antisymmetric) and free (that it is complete is obvious).
} 


\section{Definition 7 (Situation Semantics)}

$$
\begin{array}{ll}
\left\langle\left\langle R x_{1} \ldots x_{n}\right\rangle\right\rangle_{M, g} & =\left\{s \mid s=_{M, g} R x_{1} \ldots x_{n}\right\} \\
\langle\langle\neg \phi\rangle\rangle_{M, g} & =\left\{s \mid\langle\langle\phi\rangle\rangle_{M, g}=\emptyset\right\} \\
\langle\langle\exists x \phi\rangle\rangle_{M, g} & =\bigcup_{d \in E}\langle\langle\phi\rangle\rangle_{M, g[x / d]} \\
\langle\langle\phi \wedge \psi\rangle\rangle_{M, g} & =\langle\phi\rangle\rangle_{M, g} \cap\langle\langle\psi\rangle\rangle_{M, g} \\
\langle\langle A(\phi)(\psi)\rangle\rangle_{M, g} & =\left\{s \mid[A]\left(\left\{s^{\prime} \mid s^{\prime} \in\langle\langle\phi\rangle\rangle_{M, g}^{m}\right\}\right)\left(\left\{s^{\prime} \mid s^{\prime} \in\langle\langle\psi\rangle\rangle_{M, g}\right\}\right)\right\}
\end{array}
$$

In order to keep matters manageable, we have adopted a most simple notion of negation. If $\phi$ is true, then $\neg \phi$ denotes the empty set of situations, and if $\phi$ is false, $\neg \phi$ denotes the full set. Thus, the interpretation of $\neg \phi$ is deprived of any particular content. (But it is, trivially, persistent.) This is not as it generally should be, but the issue is too involved to be tackled here (see [Kratzer 1989, p. 643ff] for some relevant discussion). The same holds mutatis mutandis for the sets of situations described by adverbially quantified statements Truth-conditionally, however, they quantify over minimal situations of certain kinds. As can be seen from the definition, for a sentence If a cat falls down, it always rains to be true it must rain in all minimal situations in which a cat falls down. That is to say, for $A(\exists x(C x \wedge F x))(R)$ to be true all minimal situations $s$ which verify $\exists x(C x \wedge F x)$ must be such that $s$ verifies $R$.

In the literature on the subject one can find many suggestions about how situation structures are individuated, and this is of course relevant for the question how quantification over situations relates to quantification over cases. For instance, when Lappin and Francez in a recent paper talk about the set of minimal situations in the interpretation of $A$ man owns a donkey, they have it that: "When this set defines the range of the adverbial quantifier, the quantifier effectively quantifies over pairs which consist of a man and a donkey that he owns." ([Lappin and Francez 1994, p. 420-1]) [Heim 1990] holds more in general that: "By restricting the QAdverb's range to situations that are minimal, we have made sure that there are always just as many situations in this range as there are $n$-tuples of the relevant sort." (p. 149) Chierchia, more tentatively, suggests that we might have to conclude that "(...) occasions have to be (...) structured (..) [p]erhaps as fine grained as assignments to sequences of variables, which is of course the idea that DRT is built on." [Chierchia 1988, p. 72] Now it may be conceded that more or less intuitive assumptions about how structures of situations individuate are at stake here, but it is typical that they are never backed up by any formal constraints which guarantee that situations individuate thus. As Kadmon already had put it: "Saying something about situations or events is not in itself a solution, unless you specify how the situations/events are individuated and how that relates to the data you are trying to account for." [Kadmon 1990, p. 327]

For this reason, it is our objective to clarify this matter by investigating how the situation based domains of quantification can be constrained so as to mimic domains of $B V A$-cases. Thus, we may see what is required to get the suggested effects, and, more importantly, whether it is at all desirable to get these effects.

\section{An $S b$-WAY of GetTing $B V A$-Results}

In order to approximate the $B V A$ to adverbial quantification, the $S b A$ structure of situations has to be constrained further. If a situation semantics is appropriate for an analysis of quantifying adverbs along the above lines, and if it has to get us the $B V A$-results, it must always assign sentences propositions (sets of situations) with two properties, which are called 'minimality' and 'distinctness'.

Adverbs crucially refer to the minimal situations in the propositions (sets of situations) which are expressed by the sentences they range over ('minimality', cf., Berman 1987, Heim 1990 and von Fintel 1995, among others). Moreover, the number of minimal situations supporting the truth of a sentence must be equal to or approach the number of cases verifying it ('distinctness', cf., e.g., Heim 1990, 
the remarks on pp. 146-9). Clearly, we don't get these properties for free. For instance, even if we have a Kratzerian 巨-persistent proposition (set of situations), this does not mean that the set has smallest, or minimal, 巨-elements.And nobody sofar has excluded that a minimal situation in which, for instance, Chris smells a bargain, is also a minimal situation in which, say, cat Possum lands on its feet. Therefore we will now set ourselves the task of constraining the situation structure to get the required effects.

It may have to be said from the outset that we will assume the domains of adverbial quantification to be delineated by formulas which are free of negations and adverbial quantifiers. Like we said above, it is a relatively complicated issue to give a compositional account of the situational content of negations and adverbially quantified expressions, and in order not to distract too much from the main issue, viz., the description of case-like situations with existentially quantified formulas, we leave the matter for further study. So, henceforth, we will be dealing with formulas built up from atomic formulas using existential quantification and conjunction only.

First minimality has to be guaranteed for the propositions expressed by true atomic formulas. Such propositions must have a minimal element and this minimal element must be unique: an atomic formula can at most describe one case. The first part of the story can be captured by associating any true atomic formula $A t$ with a particular fact in $S$, which we will assume to be determined by a derived interpretation function $F_{M}{ }^{S}$ :

Definition 8 (Uniqueness) $R x_{1} \ldots x_{n}$ denotes at most one situation relative to $M$ and $g$; iff $\left\langle g\left(x_{1}\right), \ldots, g\left(x_{n}\right)\right\rangle \in F_{M}(R)$, then $R x_{1} \ldots x_{n}$ denotes a situation relative to $M$ and $g$, which is given by $F_{M} S(R)\left(\left\langle g\left(x_{1}\right), \ldots, g\left(x_{n}\right)\right\rangle\right)$

In other words, $F_{M}{ }^{S}$ is an interpretation function for the relational constants of our language which assigns to every $n$-ary constant a partial function from $n$-tuples of individuals to situations. The function is (partly) determined by our earlier models to the effect that $F_{M}{ }^{S}(R)$ is defined for a certain tuple of individuals iff the tuple of individuals is in the extension of $R$ according to $F_{M}$.

The need of uniqueness in the present context must be obvious. If we consider a model in which there is only one man, $d$, then a formula $\exists x M x$ will be $B V A$-verified by one case, viz., $d$ itself. In that case it is uniqueness that makes sure there is also (exactly) one minimal situation which $S b A$-satisfies $\exists x M x$.

With uniqueness we find that, if $R x_{1} \ldots x_{n}$ is true in $M$ under $g$, then $\left\langle\left\langle R x_{1} \ldots x_{n}\right\rangle\right\rangle_{M, g}$ is a persistent proposition $p=\left\{s^{\prime} \mid s \leq s^{\prime}\right\}$ for some unique minimal situation $s$. Using summation we also know that if $P$ and $Q$ are persistent propositions with sets of minimal elements $P^{m}$ and $Q^{m}$, then $P \cap Q$ is the persistent 巨-closure of $\left\{\left(p^{\prime} \oplus q^{\prime}\right) \mid p^{\prime} \in P^{m}\right.$ and $\left.q^{\prime} \in Q^{m}\right\}$. Summation does not by itself guarantee that such a set has minimal elements. ${ }^{2}$ However, if we are dealing with persistent propositions which have non-overlapping minimal situations ('relativized atoms'), then minimality is preserved under intersection. Such relativized atoms are given to us by the constraint to be discussed next.

The second requirement we have to impose on our situation-theoretic models is called 'distinctness'. Different atomic formulas must be guaranteed to be associated with distinct atoms. In short, it is required that:

\footnotetext{
${ }^{2}$ For instance, let, for a natural number $n, X^{n}$ be the set of positive integers $n^{\prime} \geq 2 n$ plus the negative even integers $0 \geq n^{\prime} \geq-2 n$, and $X_{n}$ the set of negative integers $n^{\prime} \leq-2 n$ plus the positive even integers $0 \leq n^{\prime} \leq 2 n$; let $P$ be the set of sets $X^{n}$ for natural numbers $n, Q$ the set of sets $X_{m}$ for natural numbers $m$; let $P^{+}$and $Q^{+}$be the closure of $P$ and $Q$ under supersets of integers, and let $\sqsubseteq$ be $\subseteq$. Then $P^{+}$is a persistent set with minimal elements $P$, similarly for $Q^{+}$and $Q$. However, the intersection of the two has no minimal elements.
} 
Definition 9 (Distinctness)

If $F_{M}{ }^{S}(R)\left(\left\langle d_{1}, \ldots, d_{n}\right\rangle\right) O F_{M}{ }^{S}(T)\left(\left\langle d_{n+1}, \ldots d_{n+m},\right\rangle\right)$, then $n=m, R=T$ and $d_{1}=d_{n+1}, \ldots$, and $d_{n}=d_{n+m}$

Distinct atomic propositions are required to be satisfied by distinct minimal siutations, where distinct means non-overlapping, not merely being different. Suppose there are two men in our model $M$, say $d$ and $d^{\prime}$, that is, there are two cases satisfying the clause 'There is a man', $\exists x M x$. Distinctness tells us in that case that there are also two minimal situations in $\left\langle\langle\exists x M x\rangle_{M}\right.$, one in which $d$ is a man, another in which $d^{\prime}$ is a man. Freedom guarantees that distinctness is preserved under conjunction. For instance, consider a model in which two different $d$ and $d^{\prime}$ are men who walk, so we have two cases $B V A$-satisfying $\exists x(M x \wedge W x)$. By uniqueness and distinctness we then have minimal and non-overlapping situations $\langle\langle M x\rangle\rangle_{g[x / d]},\langle\langle M x\rangle\rangle_{g\left[x / d^{\prime}\right]},\left\langle\langle W x\rangle_{g[x / d]},\left\langle\langle W x\rangle_{g\left[x / d^{\prime}\right]}\right.\right.$, (indicated as $M d$, $M d^{\prime}, W d$ and $W d^{\prime}$, respectively). By summation we know there are at most two minimal situations satisfying $\exists x(M x \wedge W x)$, viz., $M d \oplus W d$ and $M d^{\prime} \oplus W d^{\prime}$, and by distinctness and freedom $M d \oplus W d$ and $M d^{\prime} \oplus W d^{\prime}$ are known to be distinct, too. So we find there to be two minimal $S b A$-situations corresponding to our two $B V A$-walking men.

Notice that it would not be enough to require atomic formulas to be satisfied by different, and not merely distinct, minimal situations. For in that case we could have four situations $s, s^{\prime}, s^{\prime \prime}$ and $s^{\prime \prime \prime}$ such that $s \oplus s^{\prime}$ is a minimal situation in which $d$ is a man, $s^{\prime} \oplus s^{\prime \prime}$ one in which $d^{\prime}$ is a man, $s^{\prime \prime} \oplus s^{\prime \prime \prime}$ one in which $d$ walks, and $s^{\prime \prime \prime} \oplus s$ one in which $d^{\prime}$ walks. In that case we would have two walking men (two cases $B V A$-verifying the clause 'A man walks'), but only one corresponding situation. For if $(M d \oplus W d)=\left(\left(s \oplus s^{\prime}\right) \oplus\left(s^{\prime \prime} \oplus s^{\prime \prime \prime}\right)\right)$, it equals $\left(\left(s^{\prime} \oplus s^{\prime \prime}\right) \oplus\left(s^{\prime \prime \prime} \oplus s\right)\right)$ which is supposed to be $\left(M d^{\prime} \oplus W d^{\prime}\right)$.

We are now in a position to show that minimality is preserved under conjunction of propositions which have distinct minimal situations. Let $P^{m}$, and $Q^{m}$ be the minimal situations of persistent propositions $P$ and $Q$, respectively, and assume no two of these minimal situations overlap. Then for any $p \in P^{m}$, $q \in Q^{m}:$

Observation 6 either $p$ or $q$ is in $(P \cap Q)^{m}$, or $(p \oplus q)$ is in $(P \cap Q)^{m}$.

Proof. For any $r \in(P \cap Q)$, there are minimal $p \in P^{m}$ and $q \in Q^{m}$ such that $(p \oplus q) \sqsubseteq r$. Also, for $p \in P^{m}$ and $q \in Q^{m}$, by persistence $(p \oplus q) \in(P \cap Q)$. Now suppose $p \oplus q$ is not a minimal situation in $P \cap Q$. Then there are $p^{\prime} \in P^{m}$ and $q^{\prime} \in Q^{m}$ such that $p^{\prime} \oplus q^{\prime} \sqsubset p \oplus q$. By distinctness $\neg\left(p^{\prime} O p\right)$ or $\neg\left(q^{\prime} O q\right)$. Assume the second. Since $q^{\prime} \sqsubseteq\left(p^{\prime} \oplus q^{\prime}\right) \sqsubseteq(p \oplus q)$ freedom gives us that $q^{\prime}$ overlaps $p$ or $q$. By assumption $\neg\left(q^{\prime} O q\right)$, so $q^{\prime} O p$, whence (distinctness) $q^{\prime}=p$, so $q^{\prime}=p \in(P \cap Q)^{m}$. Analogously, we find that $p^{\prime}=q \in(P \cap Q)^{m}$ if $\neg\left(p^{\prime} O p\right)$. End of proof. More in particular we find that for some set $\left\{A t_{c} \mid c \in C\right\}$ of atomic formulas, the conjunction $\bigwedge_{c \in C} A t_{c}$ denotes a proposition with a smallest element which is the sum of the smallest elements in the denotation of the individual atomic formulas. Let, for $c \in C,\left\langle\left\langle A t_{c}\right\rangle\right\rangle_{M, h}^{m}=s_{c}^{m}$, then $\left\langle\left\langle\bigwedge_{c \in C} A t_{c}\right\rangle\right\rangle_{M, h}^{m}=\bigoplus_{c \in C} s_{c}^{m}$.

In order to get our intended result we are in need of one more constraint, one on the class of formulas to be considered. The constraint requires that if any variable is quantified over in the restrictrion $\phi$ of an adverb, then something unique is predicated of it:

Definition 10 (Unique Predication) for any variable $x$ quantified over in $\phi$, there is an atom $R x_{1} \ldots x_{n}$ in $\phi$ such that $x=x_{i}$, and for all atoms $R y_{1} \ldots y_{n}$ in $\phi: y_{i}=x$ 
It may appear as if this constraint is needed to solve the 'indistinguishable participants' (the 'cardinality problem'), but it is not really. In the set up sofar, if cardinal $c$ as a matter of fact meets cardinal $c^{\prime}$, and if by, for instance, a symmetry postulate on the predicate meet, we find that then $c^{\prime}$ also meets $c$, then distinctness still requires there to be two different minimal situations verifying the two meetings. (Clearly this may conflict with intuition, and we will therefore return to issues like this in due course.)

To see what our unique predication constraint must achieve, suppose there are two $G^{\prime} s, d$ and $d^{\prime}$. Then there are four cases verifying the formula $\exists x G x \wedge \exists y G y$ according to the $B V A$, viz. (i) $\langle d, d\rangle$, (ii) $\left\langle d, d^{\prime}\right\rangle$, (iii) $\left\langle d^{\prime}, d\right\rangle$, and (iv) $\left\langle d^{\prime}, d^{\prime}\right\rangle$. However, in a situation-based framework we find that only two minimal situations correspond to these four cases, viz., $F_{M}{ }^{S}(G)(d)$, and $F_{M}{ }^{S}(G)\left(d^{\prime}\right)$. Using $X x$ again to indicate the situation $F_{M} S(X)(x)$, it is easily seen that $G d(=(G d \oplus G d))$ corresponds to (i), that $G d^{\prime}\left(=\left(G d^{\prime} \oplus G d^{\prime}\right)\right)$ corresponds to $(i v)$, and that $\left(G d \oplus G d^{\prime}\right)=\left(G d^{\prime} \oplus G d\right)$, which corresponds to both (ii) and (iii), has both $G d$ and $G d^{\prime}$ as a proper part.

The unique predication constraint rules out this case. Consider an extension of the above formula which does satisfy the constraint, an extension with, for instance, predications $F x$ of $x$ and $H y$ of $y$, as in $\exists x(F x \wedge G x) \wedge \exists y(G y \wedge H y)$. Let us, furthermore, assume that $d$ and $d^{\prime}$ in fact are $F$ and $H$. The four cases above then correspond to the following four minimal situations: (i) $\bigoplus\{F d, G d, H d\}$, (ii) $\bigoplus\left\{F d, G d, G d^{\prime}, H d^{\prime}\right\}$, (iii) $\bigoplus\left\{F d^{\prime}, G d^{\prime}, G d, H d\right\}$, (iv) $\bigoplus\left\{F d^{\prime}, G d^{\prime}, H d^{\prime}\right\}$. By distinctness and freedom these are four different situations, as required.

Notice that if something uniquely is predicated of a variable $x$ in a conjunction $\bigwedge_{c \in C} A t_{c}$ of atomic formulas, and if for two assignments $h$ and $h^{\prime}, h(x) \neq h^{\prime}(x)$, then there is a minimal situation $\left\langle\left\langle A t_{c}\right\rangle\right\rangle_{M, h^{\prime}}^{m}$ for some $c \in C$ which is not in $\left\{\left\langle\left\langle A t_{c}\right\rangle\right\rangle_{M, h}^{m} \mid c \in C\right\}$. For let $A t_{c}$ be the unique predication of $x$, and compare, for any $c^{\prime} \in C:\left\langle\left\langle A t_{c}\right\rangle\right\rangle_{M, h^{\prime}}^{m}$ and $\left\langle\left\langle A t_{c^{\prime}}\right\rangle\right\rangle_{M, h}^{m}$. If they are the same, then, by distinctness, $A t_{c}$ and $A t_{c^{\prime}}$ must be composed with the same relational constant, and, by unique predication, the variable $x$ occupies the same distinguished position in $A t_{c}$ and $A t_{c^{\prime}}$. But this cannot be, since $h(x) \neq h^{\prime}(x)$, so $\left\langle\left\langle A t_{c}\right\rangle\right\rangle_{M, h^{\prime}}^{m}$ is not equal to $\left\langle\left\langle A t_{c}^{\prime}\right\rangle\right\rangle_{M, h}^{m}$ by distinctness.

It is finally time to come to our main result. Let $\phi$ be a formula constructed from atoms, $\exists$ and $\wedge$, which satisfies the unique predication constraint; furthermore, assume $\phi$ is $B V A$-defined for assignment $i$; finally, assume our structure to observe the constraints of summation and freedom, and our model the constraints of uniqueness and distinctness. Then, for $g$ any total extension of $i$ :

Observation 7 there is a bijection $\rho$ from $\llbracket \phi \rrbracket_{M, i}$ to $\langle\langle\phi\rangle\rangle_{M, g}^{m}$

Proof. First observe that $\phi$ has a ( $B V A$ - and $S b A$-equivalent) quantified normal form $q n f(\phi)$, which can be obtained by preposing all existential quantifiers in $\phi$, and which existentially quantifies over a (finite) set of variables in the conjunction of a (finite) set of atomic formulas: $q n f(\phi)=\exists x_{1} \ldots x_{n} \bigwedge_{c \in C} A t_{c}$, with $x_{1} \ldots x_{n}$ the variables quantified over in $\phi$ and $\left\{A t_{c} \mid c \in C\right\}$ the set of atomic formulas in $\phi$. The function $\rho$ from $\llbracket \phi \rrbracket_{M, i}$ to $\langle\langle\phi\rangle\rangle_{M, g}^{m}$ then can be defined such that for $j \in \llbracket \phi \rrbracket_{M, i}: \rho(j)=\bigoplus_{c \in C}\left\langle\left\langle A t_{c}\right\rangle\right\rangle_{M, h}^{m}$, with $h$ an arbitrary total extension of $j$. Now it remains to be shown that for any $j$ in $\llbracket \phi \rrbracket_{M, i}$ : (a) $\rho(j) \in\langle\langle\phi\rangle\rangle_{M, g}^{m}$, and that for any $s$ in $\langle\langle\phi\rangle\rangle_{M, g}^{m}$ : (b) there is (c) a unique assignment $j$ in $\llbracket \phi \rrbracket_{M, i}$ such that $\rho(j)=s$.

(a) Let $j \in \llbracket \phi \rrbracket_{M, i}$, and $h$ be a total extension of $j$. Then $i \leq_{x_{1}, \ldots x_{n}} j$ and $j \in \llbracket A t_{c} \rrbracket_{M, j}$ for all $c \in C$. Thus, for any $c \in C$ there is an atom $s_{c}^{m}$ in $\left\langle\left\langle A t_{c}\right\rangle_{M, h}\right.$, and $\rho(j)=\bigoplus_{c \in C} s_{c}^{m}$ is the smallest $s$ in $\left\langle\left\langle\bigwedge_{c \in C} A t_{c}\right\rangle\right\rangle_{M, h}$. Now suppose for some different $h^{\prime}$, which at most differs from $h$ with respect to the values of $x_{1}, \ldots x_{n}$, that for all $c \in C:\left\langle\left\langle A t_{c}\right\rangle_{M, h}\right.$ is not empty. Using unique predication and distinctness, there is a minimal situation $\left\langle\left\langle A t_{c}\right\rangle\right\rangle_{M, h^{\prime}}^{m}$ for some $c \in C$ which is not in $\left\{\left\langle\left\langle A t_{c}\right\rangle\right\rangle_{M, h}^{m} \mid c \in C\right\}$. By distinctness and freedom, we find that $\bigoplus_{c \in C}\left\langle\left\langle A t_{c}\right\rangle\right\rangle_{M, h^{\prime}}^{m} \not \subset \bigoplus_{c \in C}\left\langle\left\langle A t_{c}\right\rangle\right\rangle_{M, h}^{m}$. Therefore, $\rho(j)=\bigoplus_{c \in C} s_{c}^{m}$ is also a smallest situation in $\left\langle\left\langle\exists x_{1} \ldots x_{n} \bigwedge_{c \in C} A t_{c}\right\rangle\right\rangle_{M, g}$. 
(b) Now let $s \in\langle\langle\phi\rangle\rangle_{M, g}^{m}$. So, for some sequence of individuals $d_{1}, \ldots, d_{n}$, there is a total assignment $h=g\left[x_{1} / d_{1}\right] \ldots\left[x_{n} / d_{n}\right]$ and $s \in\left\langle\left\langle\bigwedge_{c \in C} A t_{c}\right\rangle\right\rangle_{M, h}^{m}=\left\{\bigoplus_{c \in C}\left\langle\left\langle A t_{c}\right\rangle\right\rangle_{M, h}^{m}\right\}$. But then we find for partial assignment $j=i \cup\left\{\left\langle x_{i}, d_{i}\right\rangle \mid 0<i \leq n\right\}$ that $j \in \llbracket A t_{c} \rrbracket_{M, j}$ for any $c \in C$, so $j \in \llbracket \phi \rrbracket_{M, i}$, and $\rho(j)=s$.

(c) Now let $j, j^{\prime} \in \llbracket \phi \rrbracket_{M, i}, j \neq j^{\prime}$, and $g$ and $g^{\prime}$ total extensions of $j$ and $j^{\prime}$ respectively. Then there is at least one variable $x$ such that $j(x) \neq j^{\prime}(x)$ and such that $x$ is existentially quantified over in $\phi$. By unique predication and distinctness there is an atomic formula $R x_{1} \ldots x_{n}$ in $\phi$ such that $x_{i}=x$ and such that $\left\langle\left\langle R x_{1} \ldots x_{n}\right\rangle\right\rangle_{M, g}^{m}$ is not in $\left\{\left\langle\left\langle A t_{c}\right\rangle\right\rangle_{M, g^{\prime}} \mid c \in C\right\}$. So, $\left\{\left\langle\left\langle A t_{c}\right\rangle\right\rangle_{M, g} \mid c \in C\right\} \neq\left\{\left\langle\left\langle A t_{c}\right\rangle\right\rangle_{M, g^{\prime}} \mid\right.$ $c \in C\}$, and, by freedom and distinctness, $\rho(j)=\bigoplus_{c \in C}\left\langle\left\langle A t_{c}\right\rangle\right\rangle_{M, g} \neq \bigoplus_{c \in C}\left\langle\left\langle A t_{c}\right\rangle\right\rangle_{M, g^{\prime}}=\rho\left(j^{\prime}\right)$. End of proof.

Again restricting ourselves to the quantifiers that satisfy the constraints of conservativity and quantity, and under the conditions specified above, the latest result tells us that quantification over (minimal) situations amounts to quantification over (partial) assignments. As we have seen, quantification over (partial) assignments in its turn amounts to quantification over cases. So, also quantification over (minimal) situations does. After a long road we are home.

Before we conclude this section one more observation is in order. Apparently, for a given language and model we can restrict attention to a sub-structure of situations which is the sum closure of the set of denotations of atomic formulas ('atoms'). Clearly, the resulting 'describable' structure is atomic, free, and generated, quite a delineated structure indeed. However, the present general setting shows that we are not forced to such an ontologically restricted universe. The above results can also be obtained in wider structures, that is, in less restricted extensions of describable structures. Without any danger of losing our main result, there may happen to be situations below the 'describable' structure (parts of 'atoms'), or in between it (sums of parts of 'atoms'), and also above it (non-generated situations). In other words, we do not really need to impose unrestricted summation and freedom. Summation and freedom can be restricted to hold for the describable part only.

\section{Discussion}

Sofar we have been mainly concerned with the question what it takes to get $B V A$ results on adverbial quantification in a situation-based framework. Some of the proposed constraints are relatively unobjectionable or harmless. For instance, we have excluded from consideration individual constants for the sake of simplicity. The interpretation of anaphoric relationships (dynamic binding) is put aside, because pronouns are judged of no immediate importance for the topics discussed here. (Besides, their (situation-based, bound/free variable, or otherwise) interpretation remains a matter of ongoing debate - certainly in corelation with (adverbial) quantification.) The restriction to Heimian felicity, furthermore, is independently motivated (cf., also, [Dekker 1995]). The assumption that situation structures observe the constraints of summation and freedom (or some suitably restricted variants thereof), appears to be intuitively motivated, too.

The remaining constraints are less unproblematic. For instance, it remains an open question what a notion of situation-based negation or adverbially quantification would have to be like, to give some substance to the idea that negated and adverbially quantified formulas, too, come with some specific situational content. For this purpose maybe structures of situations are needed which resemble those of Barwise and Perry's situation semantics or Landman's data semantics ([Barwise and Perry 1983, Landman 1986]). However, like we said above, this matter is left open for further research. Furthermore, our 'unique predication constraint' is too peculiar to be intuitively motivated. Yet, for the very same reason, it is also hard to argue against. This issue, then, will be left here, too.

The remaining constraints relate to the structure of situations. As we have seen, uniqueness is needed to get at least some domain of adverbial quantification, and it is related both to the minimality constraints which come with the $S b A$, as well as to Kratzer's persistence requirement. Distinctness is needed to get enough structure on the domain to mimic the $B V A$. As we will argue in the remainder 
of this paper, these constraints are at odds with relatively intuitive conceptions of situations, and this can be taken to mean that the $S b A$ and the $B V A$ are bound to make different predictions.

If it is indeed $u n$-desirable to add the constraints of uniqueness and distinctness to the situationtheoretic framework, so if these do need not to be observed, it follows that (quantification over) the domains of cases are going to differ from (quantification over) the corresponding domains of situations. For this reason we will investigate some cases where the two approaches may be expected to make different predictions. The debated constraints here may serve as heuristics for finding such cases. As we will see, it will then turn out that as a matter of fact both the $B V A$ and the $S b A$, as they have been presented in this paper, fail in some respects respects. Eventually, we will argue for a combined approach, which employs a slightly different conception of the notion of a situation.

\section{Uniqueness and Persistence}

Is it desirable at all to impose uniqueness on our situation-theoretic models, and do we want to preserve persistence? The real question here is whether we can think of there being more than one (minimal) situation in which a given tuple of individuals stands in a certain relation, and of there being one minimal situation in which a tuple of individuals does, and another in which it doesn't stand in that relation. Clearly this is possible, as soon as we take a temporal component into account. ${ }^{3}$ For, by a shift in time, the truth value of a sentence may shift from true to false, and back again, and the stretches or points of time where sentences are true may be adverbially quantified over. Consider:

(9) John always washes the dishes when Mary is asleep.

(10) When John is in bath he always sings.

The seemingly singular proposition expressed by 'John washes the dishes' or 'John is in bath' may be true of different situations (or time intervals), and these are what the adverb always in these two examples seems to quantify over. This is not really a new observation. Adverbs of quantification have been taken to range over temporal locations (time points, or intervals) in the first place, and this idea has been extensively elaborated in, among many others, [Heinämäki 1974, Stump 1981, van Eynde 1987, Chierchia 1988, de Swart 1991]. The point, however, is that this observation clashes with uniqueness, as it has been formalized above.

Paying due attention to the temporal parameter also raises a problem with persistence. Apparently, the proposition expressed by 'John is in bath' may be true in some situations (at some time intervals), and the one expressed by 'John is not in bath' in other ones. Now take a situation $s$ in which John is in bath, and a (later) one $s^{\prime}$ in which John isn't. What about the sum of these two situations? If $s$ is in the proposition expressed by 'John is in bath', and $s$ ' in that expressed by 'John is not in bath', then, by persistence, $s+s^{\prime}$ would be in both. But that should not be.

Two solutions to this problem may come to mind. The first, which remains closer to the spirit of Kratzerian situation semantics, consists in associating a determinate temporal location with the propositions expressed by sentences. So, if $s$ is in the proposition expressed by 'John is in bath' then somehow the temporal location, or 'temporal trace' of $s$ is part of the proposition, and similarly for $s$ ' and the proposition expressed by 'John is not in bath'. The above contradiction is thus avoided, since on this account the sum of $s$ and $s^{\prime}$ is in the proposition that John is in bath at the time of $s$ and not in bath at the time of $s^{\prime}$. The problem with this solution is that if the propositions involved are so determinate, then they cannot any longer serve to specify non-trivial domains of quantification. For instance, example (10) then would be interpreted as saying something like: "when John is in bath at

\footnotetext{
${ }^{3}$ Which Kratzer explicitly doesn't: "Time is not a concern in this paper. Let us put it aside whenever we can." [Kratzer 1989, pp. 611]. Kratzer may be fully justified in doing so, but as soon as we look at adverbial quantification we hardly are.
} 
$l$, he always sings", for some specific temporal location $l$ (like yesterday afternoon, at a quarter past four). Clearly, this is not the way to understand that example.

The other solution starts from the apparent observation that (temporal) adverbial quantifiers may abstract over the moments or intervals of time at which atomic sentences are true, so that the denotations of these sentences must be taken to somehow determine different moments or intervals. Now, if we want to keep on thinking of sentences as denoting situations, then their interpretation can be related to various verifying instances or moments of time, if we acknowledge that situations have a temporal location, or 'temporal trace' as it is also called, and if we allow for the possibility of multitudes of verifying situations. Clearly, this implies dropping uniquenes, and persistence, for that matter. Thus, for example, Sam had a bath can be taken to say that there was a situation in which Sam had a bath at some stretch of time in the past; Sam had a bath five times can be taken to say there were five situations of that kind in that period, and Sam didn't have a bath that no such situation is found there. When referring to yesterday, the last sentence can be conceived to be verified by the sum-situation of everything that happened yesterday iff Sam's having a bath is not part of it. Under such an analysis, clearly, Sam didn't have a bath may be verified by the situation yesterday, but not by the larger situation last week (if, for instance, he had a bath the day before yesterday). Exit persistence.

There are several proposals in the literature for dealing with situations with a temporal dimension along the lines sketched, and in these they are more commonly referred to as 'eventualities' (among many others, see, for instance, [Bach 1986, Link 1987, Krifka 1989, Landman 1992, Bartsch 1995]). Many of these also share the Davidsonian idea that eventualities or events are not merely parameters of interpretation, but in fact constitute proper arguments of verbs (cf., [Davidson 1967, Parsons 1990]). So, a verb like 'sleep' can be conceived of as a binary predicate between a class of eventualities called 'states' and individuals, and which holds of a state and an individual iff that state is a state of being asleep of that individual; and a verb like 'sell' is thought of as a quaternary relation which holds between events of selling, selling individuals, buying individuals, and things bought. In what follows we will also adopt this practice. (However, we will not delve into the question whether or not eventualities can be appropriately characterized as (spatio-)temporal locations.)

To conclude this discussion it may have to be observed that also the $B V A$ will be in need of adopting predicate-internal eventuality arguments. For recall that uniqueness was imposed on $S b A$-models for the purpose of getting $B V A$-results on adverbs of quantification. Now, if, as we argued, there is good reason not to impose uniqueness on the $S b A$, something in turn will have to be done to the $B V A$, in order for it not to get its original results, and for it to be equally satisfactory as the unconstrained $S b A$. Clearly, by thinking of situations or eventualities as constituting arguments of (verbal) predicates, this is in principle dealt with. However, in that case the issue of the individuation of situations will turn out to be of acute relevance for the $B V A$, too.

\section{Distinctness}

The other constraint to be critically discussed is that of distinctness. One of the most striking things is that distinctness excludes the possibility that two atomic proposition stand in a relation [Kratzer 1989] calls 'lumping'. If somebody painted an apple as part of her painting a still life, then the proposition that she painted a still life is said to lump the proposition that she painted an apple. Formally, a proposition $P$ is said to lump a proposition $Q$ in a world $w$ iff $w \in P$ and $\forall s \sqsubseteq w$ : if $s \in P$ then $s \in Q$. If we, as we do throughout this paper, neglect alternative possible worlds, it is easily seen that this definition amounts to saying that $P$ lumps $Q$ iff $P$ is non-empty and $P \subseteq Q$, and if we furthermore assume, as Kratzer does, that propositions are persistent, then $P$ is said to lump $Q$ iff $P$ is non-empty and for every $p \in P^{m}$ there is a $q \in Q^{m}$ : such that $q \sqsubseteq p$. Clearly, our distinctness constraint excludes this kind of lumping for two propositions expressed by any two different atomic formulas; furthermore freedom helps to exclude it for the propositions expressed by any two logically independent formulas 
composed from atomic formulas with $\exists$ and $\wedge$. This is problematic. Kratzer develops her situation theory to analyze counterfactual reasoning, and her lumping relation is a key notion in the ensueing analysis. So, either we have to give up this notion of lumping (and the analysis of counterfactuals, for that matter), or we will have to give up, or relativize, distinctness.

As a matter of fact, some form of non-distinctness has constituted one of the motivating reasons for the development of a situation-based account of adverbial quantification. [Berman 1987] discusses the following example:

(11) Usually, if a letter arrives for me, I am at home.

Imagine that the reporter of this sentence was always at home when a letter arrived for him, say fifty times, except this one time when sixty letters were delivered in one package. If all the other times the letters arrived one at a time, and if the adverb usually requires us to quantify over particular letter arrivals, then example (11) would turn to be false. However, most people are inclined to judge the sentence true, because it is taken to quantify over moments at which, or situations in which, letters are delivered. The idea is that the situation in which letter $a$ arrives is not different from the situation in which letter $b$ arrives if the arrivals are part of one delivery. Observe that this would have been excluded by distinctness.

Similar examples can be made at will.

(12) Usually, when a building burns down, the fire was started to collect the insurance.

Probably most people will agree that there is a reading of this example obtained by quantifying over buildings which burn down, but it seems the sentence can equally well be read as quantifying over fires, irrespective of the number of buildings burning down in these fires. So, if the sentence is about situations in which buildings burn down, and if burnings do not individuate according to the respective things which burn down, then the sentences the house burned down and the barn burned down may be verified by one and the same minimal event. Notice, that this is exactly what we wanted distinctness to exclude.

(13) Usually, if a mafioso enters my restaurant, I offer everybody a drink on the house (before I alarm the police).

(14) (According to Wierenga's report) Only in six cases in which policemen swept down a Beerhalle the owner succeeded in bribing them.

Also in these example it can be argued that what counts are enterings of one or more mafiosi (they usually come with four), and visits by the police, not individual mafiosi enterings, or singular Beerhalle enterings of members of police teams. To be sure, suppose two Beerhalle were visited by a team of three policemen each, and that in both cases the owner bribed the police. Probably, and hopefully, people then judge Wierenga's report as 'not standing in an unproblematic relation with reality'. Wierenga might try to back up his report by pointing that six individual policemen were involved in corrumping Beerhalle raids (or even six minimal groups of two policemen). But that wouldn't make example (14) true on its most likely reading.

With regard to (non-)distinctness, events or eventualities involving multitudes of individuals make up an interesting topic for further research. Suppose that this remarkable pianomover Geesink at once, and on his own carried three pianos upstairs. Then consider:

(15) Seven times a piano was carried upstairs.

(16) Three times a piano was carried upstairs. 
(17) A piano was carried upstairs.

(18) Three pianos were carried upstairs.

(19) Seven pianos were carried upstairs.

Apparently, examples (15) and (19) are not verified by the situation described, even though there are seven distincts sets of pianos, each element of each one of which was carried upstairs. Example (17) and (18) on the other hand must count as true, whereas example (16) appears to be false again. ${ }^{4}$ Apparently, the world is not seen to carve up into three piano-lifting events, when three piano are carried up at once.

One may of course object that example (16) must be held false because the phrase $n$ times effectively quantifies over stretches of time, and because the three individual events of lifting count as one since they are cotemporal. However, it appears to us that the phrases twice and $n$ times can be read in a non-temporal way, for instance, when we use Fifteen times a hit to describe a situation in which fifteen people simultaneously fire at and hit their respective targets. Notice, that such a similar splitting up of one time interval into various lifting events is not at all appropriate for the lifting case described here.

As a conclusion, it turns out that it is also undesirable to impose distinctness as a general constraint, and that non-distinctness in fact supplies good motivation for allowing adverbs to quantify over situations. However, it must be noticed again that this interest in situations cannot by itself be held against the $B V A$. For situations have for long been taken to be constituents of the kinds of cases quantified over according to the $B V A$, and, as we said above, the $B V A$ subsumes the $S b A$. Moreover, for a proper uniform and adequate treatment of pronouns and of the proportion problem, cases (which may include situations) may prove to be indispensable, cf., e.g., [Chierchia 1992, Dekker 1993, Jäger 1995].

\section{Minimality}

Also the constraint of minimality consitutes a heuristics for finding examples which serve to differentiate the $S b A$ and the $B V A$. One just has to try to think of sentences which can be predicted not to have minimal verifying situations, and then see how these behave under adverbial quantification. Examples are easily found with the aspectual Vendler classes of states and activities or processes. States in particular, and activities or processes to some level of fine-grainedness, are generally argued to have the 'subinterval' (or 'substate', or 'subevent') property. If $s$ is an interval at (state in) which Micky sleeps and $s^{\prime} \sqsubseteq s$ then $s^{\prime}$ is an interval at (state in) which Micky sleeps. And if $e$ is an event of running of Rob, then, up to some somehow to be specified level of relevance, if $e^{\prime} \sqsubseteq e$, then $e^{\prime}$ is an event of running of Rob. So much seems to be established wisdom in the field (cf., among many others, [Vendler 1957, Dowty 1979, Bach 1986, Link 1987, Krifka 1989, Parsons 1990, Meulen 1995]).

Since states and processes hardly can be taken to divide up into natural minimal units, they blatantly conflict with our minimality doctrine. Yet, we do find adverbial quantification over states and processes (or processes):

(20) Always, when John is asleep, Mary is awake.

(21) When John is walking he usually sings.

\footnotetext{
${ }^{4}$ Maybe except from the viewpoint of a financial administrator who must count additional charges for each case in which a piano gets carried upstairs (instead of, say, gets moved by builder's hoist). Still we think that in that case the administrator focuses on a stative reading of example (16), and that he observes that three different piano's are in three different (perfective) states of having been carried upstairs. Apparently, this is different from the reading which we want to address, which focuses on the event of carrying up. It may be noticed here that this difference between a copula- and a passive-reading of the item was is marked in Dutch and German. Moreover, the passive Drie keer werd er een piano naar boven gedragen is considered plainly false in Dutch in the circumstances described.
} 
Even if there are minimal bits of walk in John's way of walking, and such that every proper part of them is not any longer to be considered a real 'John-walk', still nobody will probably be inclined to hold that these are what one quantifies over with example (21). Similarly, it doesn't seem to make any good sense to understand example (20) as quantifying over minimal smallest states of John's sleep. (Notice also that it would not make any difference here if we let the quantifiers range, not over the states and processes themselves, but over the periods of time at which they are realized. The problem with minimality remains entirely the same.)

But if we do not get an individuated domain to quantify over by looking at minimal parts, then how does the domain of the stative and the processive individuate? How do we carve up the domains of states and processes, in order to 'quantize' them, as Krifka calls it? Interestingly, one plain answer to that question is by maximalization! [Parsons 1990, Kratzer 1989, Bartsch 1995] and von Fintel (in a recent eletronic exchange of findings) each stress the importance of some notion of maximality: [Parsons 1990, p. 184]: "We need only add that English usage requires that when we discuss an event that constitutes a process we usually have in mind a 'maximal' event of its kind, so that if someone asks about 'Agatha's running' we assume that the person is mentioning the whole run, not one of its parts." [Kratzer 1990, p. 5]: "Likewise we have to make a choice when talking about the facts that

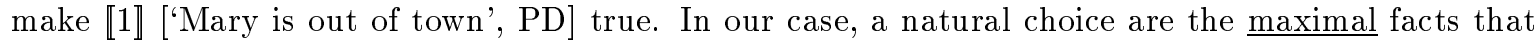

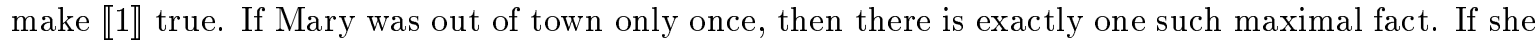

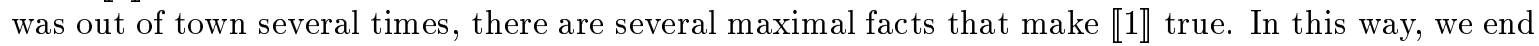
up with one fact per absence, and this is as it should be." [Bartsch 1995, p. 33] points at: "... the fact that we can only quantify over (finite) maximal states and processes and not over non-maximal ones." Von Fintel concludes his reflections: "that there is something fundamentally wrong with the minimality doctrine. ... The alternative is to figure out some way of forcing quantifiers to quantify (i) over objects at the same level of the part-whole hierarchy, (ii) over 'maximally uninterrupted' objects."

Interestingly, the present observations require us to re-do our homework. Before we even can make sense of quantification over maximal states and events, we need to be sure they exist at all, and not in a too trivial sense. To see, again, that things don't come for free, consider the kind of structures discussed by Bach and Krifka. There we find a discussion of the property of 'cumulativity' in the nominal domain, and of what Krifka calls 'summativity' in the verbal domain, two properties which involve closures under sums: every two parts of water are taken to be water together, and the sum of two events of drinking wine by you is considered a drinking wine by you. Now, if a state denoting verb has this closure property, then, strictly speaking, either there will be no maximal state, or there will only be one. Of course, none of these two 'individuations' corresponds to any intuitive one.

Clearly, we have to be more specific about 'maximal' states and processes, and think of them as states which constitute coherent wholes, for instance, as von Fintel's maximally uninterrupted objects. We think, what we really need are sensible perspectives under which 'densy' (sub-)stuctures of situations can be seen to individuate. A straightforward example of a perspective is a temporal, or a spatio-temporal one. Without going into any of the required detail, maximal states can be thought of as those states which have a connected temporal, or spatio-temporal location. Thus, the temporal location of a number of things can be used to cover distinctions, viz., between those entities which (temporally) overlap, and also to uncover distinctions, viz., among those which are (temporally) disjoint. More in general, a perspective can be thought of as a way of seeing things, which guides one in making some distinctions, and neglecting others.

There are several ways to go about here. (Cf., e.g., [Bartsch 1995], in which verbal predicates are assumed to come with their own perspectives, or 'principles of individuation' as they are called there.) However, whether it be in these or different ways, states and processes will have to be mould into some discrete form before they can be subjected to adverbial quantification. Precisely how this happens, what operations are involved, and what presuppositions the structures of states and processes must 
fulfill for the operations to be able to work on them at all, that is a matter we have to leave for another occasion.

\section{Conclusion}

In this paper we have studied the relation between the so-called situation-based and the bound variable approach to adverbial quantification. We have shown what it would take to verify the suggestion that if the individuation of situations is spelled out adequately, then the two approaches converge. We have seen that this requires us to impose three constraints on situation structures, viz., those here called uniqueness, distinctness and minimality, which one as a matter of fact should not want to impose. We have next opted for a shift of the conception of a situation to that of an eventuality, and for a treatment of the adverbs in a dynamic event-based framework.

In a sequel to this paper we would like to investigate techniques to turn (domains of) states and processes into proper subjects of quantification, and to cast these in a general theory of adverfbial quantification in a two-sorted (dynamic) predicate logical framework. Here we want to conclude while retaining our reserved attitude towards situations and events: "[f] or events and states are among the most problematic ontological categories, the identity criteria of which are difficult to apply not just in a few marginal cases, thought up by ill-meaning philosophers bent upon showing their ultimate fragility, but in perfectly ordinary, run-of-the-mill cases as well." [FraCaS 1994, p. 65] Cats are nice, indeed, especially Siamese twin cats.

\section{REFERENCES}

[Bach 1986] Emmon Bach. The algebra of events. Linguistics and Philosophy, 15:5-16, 1986.

[Bartsch 1995] Renate Bartsch. Situations, Tense and Aspect: Dynamic Discourse Ontology and the Semantic Flexibility of Temporal System in German and English. GRASS. Mouton de Gruyter, Berlin, 1995.

[Barwise and Perry 1983] Jon Barwise and John Perry. Situations and Attitudes. MIT Press, Cambridge MA, 1983.

[Berman 1987] Stephen Berman. Situation-based semantics for adverbs of quantification. In J. Blevins and A. Vainikka, editors, University of Massachusetts Occasional papers 12, pages 45-68. University of Massachusetts, Amherst, 1987.

[Chierchia 1988] Gennaro Chierchia. Dynamic generalized quantifiers and donkey anaphora. In Manfred Krifka, editor, Genericity in Natural Language, pages 53-83. SNS, Tübingen, 1988.

[Chierchia 1992] Gennaro Chierchia. Anaphora and dynamic binding. Linguistics and Philosophy, 15:111-83, 1992.

[Davidson 1967] Donald Davidson. The logical form of action sentences. In N. Rescher, editor, The Logic of Decision and Action, pages 81-95. University Press, Pittsburgh, 1967. also appeared in Donald Davidson, 1980, Essays on Actions and Events, Clarendon Press, Oxford.

[de Swart 1991] Henriëtte de Swart. Adverbs of Quantification: a Generalized Quantifier Approach. PhD thesis, Rijksuniversiteit Groningen, 1991.

[Dekker 1993] Paul Dekker. Transsentential Meditations. Ups and downs in dynamic semantics. PhD thesis, ILLC/Department of Philosophy, University of Amsterdam, Amsterdam, 1993.

[Dekker 1995] Paul Dekker. The values of variables in dynamic semantics. Linguistics and Philosophy, 19, 1995. in press.

[Dowty 1979] David Dowty. Word Meaning and Montague Grammar. Synthese Language Library. Reidel, Dordrecht, 1979.

[Fintel 1995] Kai von Fintel. A minimal theory of adverbial quantification. In Hans Kamp and Barbara Partee, editors, Context in the Analysis of Linguistic Meaning, Stuttgart/Prague, 1995. 
IMS/UFAL. draft.

[FraCaS 1994] L.R.E. FraCaS. Harmonizing the approaches. Deliverable D7, CCS, Edinburgh, December 1994.

[Groenendijk and Stokhof 1991a] Jeroen Groenendijk and Martin Stokhof. Dynamic predicate logic. Linguistics and Philosophy, 14:39-100, 1991.

[Groenendijk and Stokhof 1991b] Jeroen Groenendijk and Martin Stokhof. A note on interrogatives and adverbial quantification. In Chris Barker and David Dowty, editors, Preroceedings of SALT II, Ohio, 1991. Ohio State University.

[Heim 1982] Irene Heim. The Semantics of Definite and Indefinite Noun Phrases. PhD thesis, Amherst, MA, 1982. published 1989 by Garland.

[Heim 1990] Irene Heim. E-type pronouns and donkey anaphora. Linguistics and Philosophy, 13:137$177,1990$.

[Heinämäki 1974] Orvokki Heinämäki. Semantics of English temporal connectives. PhD thesis, University of Texas, 1974. published 1978 by the Indiana University Linguistics Club.

[Jäger 1995] Gerhard Jäger. Topics in Dynamic Semantics. PhD thesis, Humboldt Universität, Berlin, 1995.

[Kadmon 1990] Nirit Kadmon. Uniqueness. Linguistics and Philosophy, 13:273-324, 1990.

[Kratzer 1989] Angelika Kratzer. An investigation of the lumps of thought. Linguistics and Philosophy, 12:607-653, 1989.

[Kratzer 1990] Angelika Kratzer. How specific is a fact. University of Massachusetts, August 1990.

[Krifka 1989] Manfred Krifka. Nominal reference, temporal constitution, and quantification in event semantics. In Renate Bartsch, Johan van Benthem, and Peter van Emde Boas, editors, Semantics and Contextual Expression. Foris, Dordrecht, 1989.

[Landman 1986] Fred Landman. Towards a theory of information. The status of partial objects in semantics. Foris, Dordrecht, 1986.

[Landman 1989] Fred Landman. Groups. I. Linguistics and Philosophy, 12:559-605, 1989.

[Landman 1992] Fred Landman. The progressive. Natural Language Semantics, 1:1-32, 1992.

[Lappin and Francez 1994] Shalom Lappin and Nissim Francez. E-type pronouns, i-sums, and donkey anaphora. Linguistics and Philosophy, 17(4):391-428, 1994.

[Lewis 1975] David Lewis. Adverbs of quantification. In Edward L. Keenan, editor, Formal Semantics of Natural Language, pages 3-15. Cambridge University Press, Cambridge, U.K., 1975.

[Lewis 1991] David Lewis. Parts of Classes. Basil Blackwell, Oxford, 1991.

[Link 1987] Godehard Link. Algebraic semantics of event structures. In Jeroen Groenendijk, Martin Stokhof, and Frank Veltman, editors, Proceedings of the Sixth Amsterdam Colloquium, Amsterdam, 1987. ITLI.

[Lønning 1996] Jan Tore Lønning. Plurals and collectivity. In Johan van Benthem and Alice ter Meulen, editors, Handbook of Logic and Language. Elsevier, 1996. in press.

[Meulen 1995] Alice ter Meulen. Representing Time in Natural Language: the Dynamic Interpretation of Tense and Aspect. MIT Press, Cambridge (MA), 1995.

[Milward 1996] David Milward. Integrating situations into a theory of discourse anaphora. In Paul Dekker and Martin Stokhof, editors, Proceedings of the Tenth Amsterdam Colloquium, Amsterdam, 1996. ILLC.

[Parsons 1990] Terence Parsons. Events in the Semantics of English. MIT Press, Cambridge, MA, 1990. 
[Root 1986] Rebecca Root. The Semantics of Anaphora in Discourse. PhD thesis, University of Texas, Austin, 1986.

[Simons 1987] Peter Simons. Parts. A Study in Ontology. Clarendon Press, Oxford, 1987.

[Stump 1981] Greg Stump. The Formal Semantics and Pragmatics of Free Adjuncts and Absolutes in English. PhD thesis, Ohio State University, Ohio, 1981.

[Tarski 1956] Alfred Tarski. Foundations of the geometry of solids. In Alfred Tarski, editor, Logic, Semantics, Metamathematics. Papers from 1923 to 1938, chapter II. Clarendon Press, Oxford, 1956. an earlier version was published in 1929 under the title "Les Fondements de la Géométrie des Corps" in Księga Pamiątkowa Pierwszego Polskiego Zjazdu Matematyczkego, Supplement to Annales de la Société Polonaise de Mathématique, Kraków, p. 29-33.

[van Benthem 1983] Johan van Benthem. The Logic of Time. A Model-theoretic Investigation into the Varieties of Temporal Ontology and Temporal Discourse. Reidel, Dordrecht, 1983.

[van Eijck and de Vries 1991] Jan van Eijck and Fer-Jan de Vries. Dynamic interpretation and Hoare deduction. Journal of Logic, Language and Information, 1:1-44, 1991.

[van Eynde 1987] Frank van Eynde. Iteration and quantification. In Jeroen Groenendijk, MArtin Stokhof, and Frank Veltman, editors, Proceedings of the Sixth Amsterdam Colloquium, Dordrecht, 1987. ITLI.

[Vendler 1957] Zeno Vendler. Verbs and times. The Philosophical Review, 66:143-160, 1957. also published in Zeno Vendler, 1967, Linguistics and Philosophy, Cornell UP, Ithaca. 